Photonic quantum transport in a nonlinear optical fiber

This article has been downloaded from IOPscience. Please scroll down to see the full text article.

2011 EPL 9454006

(http://iopscience.iop.org/0295-5075/94/5/54006)

View the table of contents for this issue, or go to the journal homepage for more

Download details:

IP Address: 131.215.220.185

The article was downloaded on 10/06/2011 at 15:49

Please note that terms and conditions apply. 


\title{
Photonic quantum transport in a nonlinear optical fiber
}

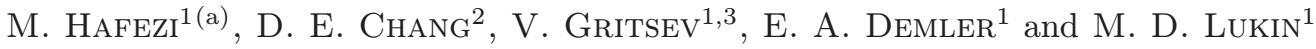 \\ ${ }^{1}$ Physics Department, Harvard University - Cambridge, MA 02138, USA \\ ${ }^{2}$ Center for the Physics of Information and Institute for Quantum Information, California Institute \\ of Technology - Pasadena, CA 91125, USA \\ ${ }^{3}$ Physics Department, University of Fribourg - Chemin du Musée 3, 1700 Fribourg, Switzerland
}

received 29 July 2010; accepted in final form 26 April 2011

published online 27 May 2011

PACS 42.65.-k- Nonlinear optics

PACS 05.60.Gg - Quantum transport

PACS 42.50.-p-Quantum optics

\begin{abstract}
We theoretically study the transmission of few-photon quantum fields through a strongly nonlinear optical medium. We develop a general approach to investigate nonequilibrium quantum transport of bosonic fields through a finite-size nonlinear medium and apply it to a recently demonstrated experimental system where cold atoms are loaded in a hollow-core optical fiber. We show that when the interaction between photons is effectively repulsive, the system acts as a single-photon switch. In the case of attractive interaction, the system can exhibit either antibunching or bunching, associated with the resonant excitation of bound states of photons by the input field. These effects can be observed by probing statistics of photons transmitted through the nonlinear fiber.
\end{abstract}

Copyright (C) EPLA, 2011

Quantum dynamics of strongly correlated systems far from equilibrium is a new frontier of many-body physics. Intriguing phenomena involving such dynamics have recently been observed in diverse physical systems ranging from ultra-cold atoms [1] to individual spins in semiconductors $[2,3]$. At the same time, recent experiments using ultra-cold atoms and optical photons [4-6] have opened the door for studies of a novel form of quantum transport involving strongly correlated photons. It has been predicted that these systems can allow for surprising behavior, such as the dynamical creation of a "crystal" of photons [7]. While many similarities exist between interacting photonic systems and condensedmatter systems involving massive particles [8-13], the photonic systems also present a unique set of challenges. In particular, they do not thermalize, and are inherently open, driven systems, which highlights the need to develop novel techniques for analysis.

In this letter, we describe a general technique to study the quantum transport of a few field quanta through a finite-length, strongly nonlinear one-dimensional waveguide. This technique allows us to determine the full

(a) Present address: Joint Quantum Institute, University of Maryland - College Park, MD 20742, USA; E-mail: hafezi@umd.edu spatial wave functions of the photons inside the waveguide as well as correlation functions of the outgoing reflected and transmitted light. We consider an optical waveguide in which the tight confinement of photons near the diffraction limit $[4,6,14,15]$ and the large number of atoms with which they interact should enable large optical nonlinearities at the single-photon level, which is necessary for applications like single-photon switching and photonic quantum gates [16-18]. Such system is in contrast to standard nonlinear optical fibers where the nonlinearity is weak and large optical intensity is needed (see, e.g., refs. $[19,20])$. Moreover, unlike nonlinear optical effects in cavity quantum electrodynamics (QED) [21], where only a single spatial cavity mode is involved, these waveguide systems are more difficult to treat in that they contain a large number of spatial degrees of freedom, much like low-dimensional, strongly interacting condensed-matter systems [22-26] containing a few quanta. As a specific application, we use the calculated reflection and transmission amplitudes to demonstrate how such a system can be used to realize a single-photon switch. Our analysis reveals the tendency for photons to "organize" themselves due to an effective tunable repulsive or attractive interaction (as shown in fig. 1). In the latter case, we show that two-photon bound states can form inside the waveguide, 


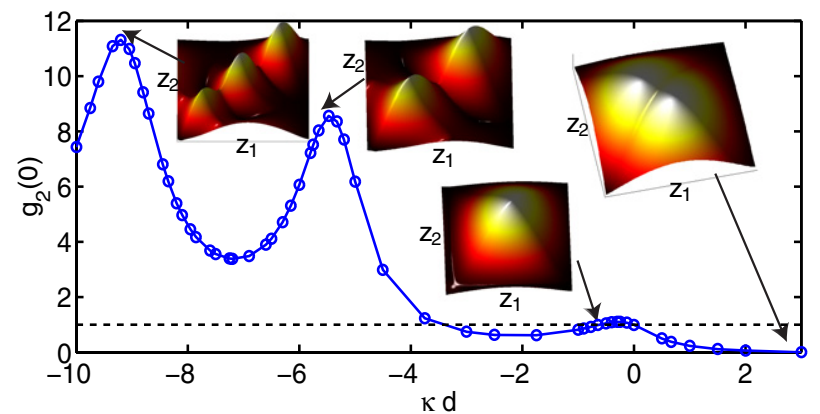

Fig. 1: (Colour on-line) Attractive $(\kappa<0)$ and repulsive $(\kappa>0)$ photons: Output correlation function $g_{2}(\tau=0)$ as a function of nonlinearity. The two-photon wave functions $\left(\left|\phi\left(z_{1}, z_{2}\right)\right|\right)$ for four values of the nonlinearity are shown. The system size is $d=30$.

which can be observed using standard quantum optical measurements of the output light.

Specifically, we are interested in the situation where the dynamics of photons inside the waveguide is governed by the quantum nonlinear Schrödinger equation (NLSE) $[7,27]$

$$
i \frac{\partial \hat{\Psi}}{\partial \tau}=-\frac{1}{2 m} \frac{\partial^{2} \hat{\Psi}}{\partial z^{2}}+2 \kappa \hat{\Psi}^{\dagger} \hat{\Psi}^{2} .
$$

Here $\hat{\Psi}$ is a photonic field annihilation operator whose specific form will be specified later. The presented framework can be used to treat the quantum transport of any NLSE system (such as interacting atoms that are constrained to move along one dimension [24,25,28]), a problem that in general remains relatively unexplored. However, we focus on a photonic system implemented recently in ref. [4], where an ensemble of atoms is loaded in a hollow-core fiber to mediate the interaction between photons. Moreover, we take advantage of quantum optical techniques $[29,30]$ based on electromagnetically induced transparency (EIT) and photon trapping which allows us to dynamically control the effective mass and interaction strength in eq. (1) [7]. The light inside the fiber interacts with an ensemble of atoms (with linear density $n_{0}$ and length $L$ ) whose level scheme is shown in fig. 2. Following refs. [7,30], we use forward- and backward-going darkstate polariton operators $\hat{\Psi}_{ \pm}$, which describe excitations of slowly-varying photonic fields $\hat{\mathcal{E}}_{ \pm}$that are coupled to an atomic spin wave excitation $\sigma_{a c}$ through a standing-wave control field $\Omega_{ \pm}(t)=\Omega$. The standing wave creates an effective Bragg grating that couples $\hat{\Psi}_{ \pm}$together and traps them inside the medium [30,31], much like an optical cavity. In the limit of large optical depth $\left(\mathrm{OD}=n_{0} L \frac{\Gamma_{1 D}}{\Gamma}\right)$, the symmetric combination $\hat{\Psi}=\left(\frac{\hat{\Psi}_{+}+\hat{\Psi}_{-}}{\sqrt{2}}\right)$ becomes the only independent quantity while the antisymmetric combination $\hat{A}=\left(\frac{\hat{\Psi}_{+}-\hat{\Psi}_{-}}{\sqrt{2}}\right)$ adiabatically follows, $\hat{A} \simeq-\frac{i}{2 m} \partial_{z} \hat{\Psi}$. The detuning $\Delta_{1}$ leads to a quadratic dispersion that can be interpreted as an effective mass $m=\frac{1}{2}\left(1+i \frac{\Gamma}{2\left|\Delta_{1}\right|}\right)$. The presence of the additional

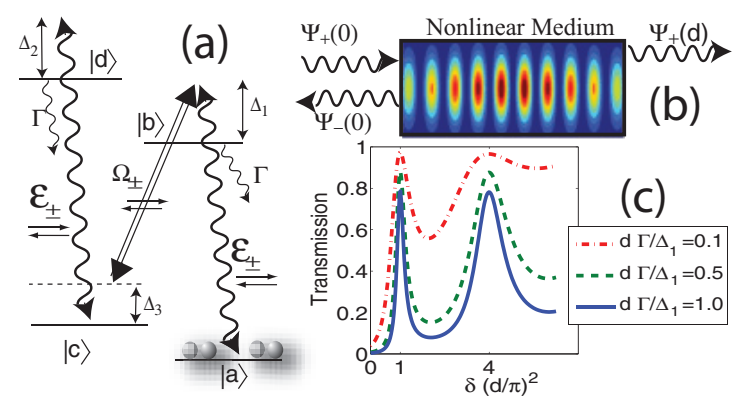

Fig. 2: (Colour on-line) (a) Four-level atomic system yields a field evolution equation in the form of an NLSE. (b) A schematic of quantum transport inside a finite-length waveguide. An input field $\hat{\Psi}_{+}(0)$ is injected into one end of the waveguide. Inside the medium, the effective Bragg grating couples the forward- and backward-propagating fields together and the evolution of the coupled fields is described by the NLSE. A field is transmitted (reflected) at the end of the waveguide $z=d(z=0)$. (c) Linear transmission spectrum $(\kappa=0)$ as a function of detuning, for a system of optical depth $\mathrm{OD} \equiv n_{0} L \frac{\Gamma_{1 D}}{\Gamma}=1000$.

atomic state $|d\rangle$ leads to an optical nonlinearity [32] whose strength is given by $\kappa=\frac{\Gamma_{1 D}}{4\left(\Delta_{2}+i \Gamma / 2\right)}$, where $\Gamma_{1 D}$ is the spontaneous emission rate into the guided modes and $\Gamma$ is the total spontaneous emission rate of both states $|b\rangle$ and $|d\rangle\left(\Gamma_{1 D} \leqslant \Gamma\right)$. We are primarily interested in the limit $\left|\Delta_{1,2}\right| \gg \Gamma$ such that $m, \kappa$ are mostly real and the evolution is dispersive. Finally, we note that eq. (1) is written in dimensionless units; all lengths and time are scaled by $L_{c o h}=2\left(\Delta_{1}^{2}+\Gamma^{2} / 4\right) / \Gamma_{1 D} n_{0}\left|\Delta_{1}\right|$ and $t_{c o h}=\left|\Delta_{1}\right| / 2 \Omega^{2}$, respectively.

First, we study the linear limit where $\kappa=0$, and the field is classical $(\Psi=\langle\hat{\Psi}\rangle)$. We investigative the linear transmission spectrum of a finite system of dimensionless length $d=\frac{L}{L_{c o h}}$, where an input field $\Psi_{+}(0)=\alpha_{0} e^{-i \delta \tau}$ is driving one end of the system and no input is at the other end $\left(\Psi_{-}(d)=0\right)$ (see fig. $\left.2(\mathrm{~b})\right)$. By solving a set of coupled mode equations for $\Psi_{ \pm}[31]$, one can show that like an optical cavity, the coupling of $\Psi_{ \pm}$inside the waveguide creates a set of transmission resonances in the output field $\Psi_{+}(d)$, which due to the quadratic dispersion of the system occurs at $\delta=\left(\frac{n \pi}{d}\right)^{2}[27]$. Here $\delta=\Delta_{3} \mathrm{t}_{\text {coh }}$ is the dimensionless two-photon detuning and $n$ is a positive integer (fig. 2(c)). For large systems $(d \gg 1)$, the effective cavity finesse scales as $\mathcal{F} \propto d^{2}$. Consequently, for a fixed resonant optical depth $\left(\mathrm{OD} \simeq d \frac{\left|\Delta_{1}\right|}{\Gamma}\right), \Delta_{1}$ can be tuned to yield either a sharp and low transmission peak (large $d$ ) or a broad and high peak ( small $d$ ), as shown in fig. 2(c). The attenuation is exponential in length and is characterized by $\beta=d \frac{\Gamma}{\left|\Delta_{1}\right|}$. Therefore, to have an effective cavity with a fixed attenuation rate, the detuning should be chosen according to $\left|\Delta_{1}\right|=d \frac{\Gamma}{\beta}$. This means that the effective finesse of the system scales as $\mathcal{F} \propto d^{2} \simeq \beta$ OD.

The finesse of this effective cavity is proportional to OD [27]. For a classical field, addition of the nonlinear 
term shifts the transmission peaks in frequency in an intensity-dependent way to the left or right depending on the sign of the nonlinearity coefficient. Transport in the classical NLSE system has already been extensively studied, where the nonlinearity is sufficiently weak that the operators in eq. (1) can be replaced by complex numbers representing their mean-field values. In the context of atomic Bose-Einstein condensate transport [33-35], this yields the well-known Gross-Pitaveskii equation, while in nonlinear optical fibers the classical NLSE explains, for instance, the formation of optical solitons [19]. While the nonlinearity in our system can be made small enough to also observe such effects, here we are primarily interested in exploring the novel regime where the nonlinearity becomes extremely strong. In particular, when $\kappa \mathrm{OD} \gg 1$, the frequency shift is predicted to be significant at intensities corresponding to a single-photon level. In this regime, the quantum transport of a few photons is expected to behave fundamentally differently than predicted by classical calculations, as we describe below.

In order to study quantum transport properties of the system, we investigate the evolution of the wave function of the field in the Schrödinger picture. We are interested in the situation where the system contains no more than a few photons, which is sufficient to describe the properties of a single-photon switch. This allows us to truncate the Hilbert space so that only vacuum, single-photon, and twophoton states are present (the analysis can be extended to truncating at any small number of photons). We thus write the general state as

$$
\begin{aligned}
|\psi(t)\rangle= & \int \mathrm{d} z_{1} \mathrm{~d} z_{2} \phi\left(z_{1}, z_{2}, t\right) \hat{\Psi}^{\dagger}\left(z_{1}\right) \hat{\Psi}^{\dagger}\left(z_{2}\right)|0\rangle \\
& +\int \mathrm{d} z \theta(z, t) \hat{\Psi}^{\dagger}(z)|0\rangle+\epsilon|0\rangle,
\end{aligned}
$$

where the amplitude of the vacuum state is close to one $(\epsilon \simeq 1)$. The first, second and third terms on the right correspond to the two-photon, single-photon and vacuum components, respectively. Importantly, directly solving for the two-photon wave function $\phi\left(z_{1}, z_{2}, t\right)$ allows us to characterize any nontrivial spatial order between two photons in the system. The evolution of the two-photon wave function $\phi\left(z_{1}, z_{2}, t\right)$ under eq. (1) is given by

$$
i \partial_{t} \phi=-\frac{1}{2 m}\left(\partial_{z_{1}}^{2}+\partial_{z_{2}}^{2}\right) \phi+2 \kappa \phi \delta\left(z_{1}-z_{2}\right),
$$

while the single-photon state evolves as a free, massive particle. Generally, direct integration of such evolution equations should be a viable approach for any transport problem involving only a few photons (or any other particles). A novel characteristic of photonic systems, however, is that light inherently can also enter and leave. Thus a remaining task is to formulate an "input-output" formalism that allows one to relate the wave functions inside the waveguide $(0 \leqslant z \leqslant d)$ to the fields injected into and propagating out from the system and any of their correlation functions, which we describe below.
Since the NLSE Hamiltonian commutes with the field number operator $\left(\hat{\Psi}^{\dagger} \hat{\Psi}\right)$, manifolds with different field quanta are decoupled from each other inside the medium. However, the system is driven with an input field at $z=0$, and different manifolds can be coupled at the boundaries. In particular, for a classical input field, the boundary conditions correspond to that of a coherent state,

$$
\hat{\Psi}_{+}(z=0)|\psi(t)\rangle=\alpha_{0} e^{-i \delta \tau}|\psi(t)\rangle,
$$

where $\alpha_{0}$ is the coherent state amplitude and $\delta$ the detuning. Since $\hat{\Psi}_{+}$annihilates a photon, the boundary condition relates any manifold with $n$ photons to that with $n-1$. Using the fact that the antisymmetric combination $\hat{A}$ adiabatically follows $\hat{\Psi}$, we can rewrite $\hat{\Psi}_{ \pm}$purely in terms of the field of interest $\Psi, \hat{\Psi}_{ \pm}=\frac{1}{\sqrt{2}}\left(\hat{\Psi} \mp i \frac{1}{2 m} \partial_{z} \hat{\Psi}\right)$. From now on in the analytical expressions, we consider the mass to be real ( $m \simeq 1 / 2$, i.e., large detunings: $\left.\Delta_{1} \gg \Gamma\right)$, while in the numerical simulations, the imaginary part is also taken into account. Therefore, the boundary condition at $z=0$ becomes:

$$
\hat{\Psi}(z=0)-i \partial_{z} \hat{\Psi}(z=0)|\psi(t)\rangle=\sqrt{2} \alpha_{0} e^{-i \delta \tau}|\psi(t)\rangle .
$$

Using the multi-mode wave function of the system from eq. (2), the boundary condition for $z=0$ then reads $\phi\left(0, z_{2}, t\right)-\left.i \partial_{z_{1}} \phi\left(z_{1}, z_{2}, t\right)\right|_{z_{1}=0}=\frac{\alpha_{0}}{\sqrt{2}} e^{-i \delta \tau} \theta\left(z_{2}, t\right)$ and $\theta(z=0, t)-i \partial_{z} \theta(z=0, t)=\sqrt{2} \alpha_{0} e^{-i \delta \tau} \epsilon$, and $\epsilon \simeq 1$. Similar boundary condition applies at $z=d$ with no input field $\left(\alpha_{0}=0\right)$ :

$$
\hat{\Psi}(z=d)+i \partial_{z} \hat{\Psi}(z=d)|\psi(t)\rangle=0
$$

The intensity or any other field correlation function can easily be obtained from the photon wave functions. For example, the normalized second-order correlation function of the output field $\hat{\Psi}_{+}(z=d)$ is given by

$$
g_{2}(0)=\frac{4|\phi(d, d)|^{2}}{\left(|\theta(d)|^{2}+4 \int \mathrm{d} z|\phi(z, d)|^{2}\right)^{2}} .
$$

Here, we have again expressed $\hat{\Psi}_{ \pm}$in terms of $\Psi$, using the relations $\hat{\Psi}_{-}|\psi\rangle=\frac{1}{\sqrt{2}}\left(\hat{\Psi}+i \partial_{z} \hat{\Psi}\right)|\psi\rangle=0$ and $\hat{\Psi}_{+}=\sqrt{2} \hat{\Psi}$ at $z=d$.

Before studying the dynamics, we analyze the fundamental modes and energy spectrum supported by the system in the absence of any input field $\left(\alpha_{0}=0\right.$ at both $z=0$ and $z=d$ ), which can be found via the Bethe ansatz technique $[22,36]$. This ansatz specifies that the eigenstates consist of a superposition of states in which colliding particles exchange their wave numbers $k_{i}$. Unlike the typical formulation, the values of $k_{i}$ here can be complex to reflect the open nature of our boundary conditions, which allow particles to freely enter or leave. For single particles, the interaction is absent and the solution takes the free propagating form: $e^{ \pm i k z}$. By applying the boundary conditions, we find that the wave number satisfies

$$
e^{2 i k d}=\left(\frac{k+1}{k-1}\right)^{2}
$$


For higher number of particles, by generalizing the Bethe ansatz and imposing the open boundary conditions, one finds that the fundamental modes satisfy

$$
e^{2 i k_{i} d}=\frac{\left(k_{i}+1\right)^{2}}{\left(k_{i}-1\right)^{2}} \prod_{i \neq j} \frac{\left(k_{i}-k_{j}+i \kappa\right)\left(k_{i}+k_{j}+i \kappa\right)}{\left(k_{i}-k_{j}-i \kappa\right)\left(k_{i}+k_{j}-i \kappa\right)} .
$$

Here, we focus on the case of two particles, such that $i, j \in$ $\{1,2\}$ and $i \neq j$ (for detailed derivation and generalization to the many-body case see ref. [27]). Correspondingly, the energy of each state $E=k_{1}^{2}+k_{2}^{2}$ can acquire an imaginary part describing its rate of exit. One class of allowed solutions corresponds to the quasi-momenta of free photons propagating in our open finite system. In the strong-interaction limit, these wave vectors take on the allowed values for the noninteracting case, regardless of whether the interaction is attractive $(\kappa>0)$ or repulsive $(\kappa<0)$. In the strongly repulsive case $(\kappa d \gg 1)$, this phenomenon is well-known as the fermionization of hardcore bosons [28]. We note that the parameter $\kappa d$ is similar to the ratio of the interaction to kinetic energy in the context of a Tonks-Girardeau gas [37]. In the strongly attracting case, these states are known as a superTonks gas $[38,39]$. For a large system $(d \gg 1)$, the noninteracting wave vectors and energy can be approximated by $\left(\frac{m \pi}{d}, \frac{n \pi}{d}\right)$ and $\left(\frac{n \pi}{d}\right)^{2}+\left(\frac{m \pi}{d}\right)^{2}$, respectively. A second class of solutions appears for negative $\kappa$ and corresponds to bound states whose momenta for large system size can be approximated by $\left(k_{1}, k_{2}\right) \simeq \frac{n \pi}{d} \pm i \frac{\kappa}{2}$. In other words, the interaction Hamiltonian $V=-2|\kappa| \delta\left(z_{1}-z_{2}\right)$ admits a single bound state in the relative coordinate of the two photons, while the allowed momenta in the center-of-mass coordinates are quantized and determined by the system size. This yields a discrete set of bound state energies $E_{n}^{b} \simeq 2\left(\frac{n \pi}{d}\right)^{2}-\frac{\kappa^{2}}{2}$.

We now examine the properties of the light transmitted through the waveguide, first considering the repulsive regime $\kappa>0$, which corresponds to $\Delta_{2}>0$. We fix the input field detuning to $\delta_{\text {res }}=(\pi / d)^{2}$, which corresponds to the first transmission resonance in the linear regime. Since we wish to study an open, driven system out of equilibrium, conventional analytical methods such as Bethe ansatz or quantum inverse scattering [23] cannot be applied here, whereas eq. (3) with proper boundary conditions can easily be numerically solved. We assume that the photonic system is initially in the vacuum state. After a time roughly equal to the inverse of the system bandwidth $\left(\simeq d^{2}\right)$, the system reaches a steady state, and we present stationary transmission coefficients and correlation functions in this limit. Although all numerical results correspond to a specific set of parameters (system size, detuning, etc.), the conclusions are quite general. In the presence of linear absorption, only a fraction of the singlephoton state is transmitted and the rest is dissipated $(T<1)$; this results in the imperfect transmission shown in fig. 2(c), since the single-photon state obeys linear dynamics. We note that since the system is weakly driven

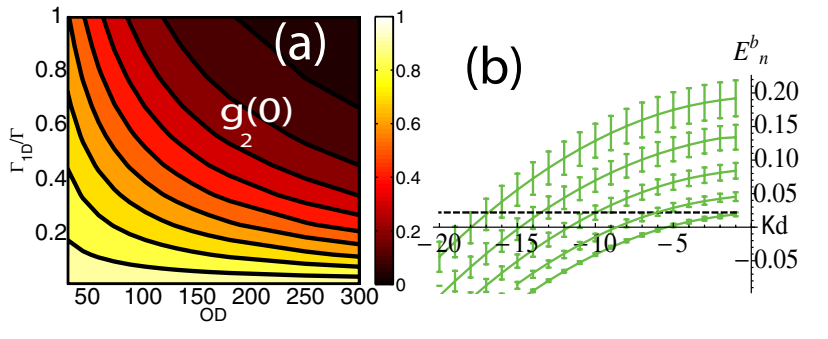

Fig. 3: (Colour on-line) (a) Repulsive photons: correlation function $g_{2}(\tau=0)$ of the transmitted light as a function of optical depth $\left(\mathrm{OD}=n_{0} L \frac{\Gamma_{1 D}}{\Gamma}\right)$ and single-atom cooperativity when the frequency is set to the single-photon transmission resonance with $T \simeq 20 \%$ and $\frac{\Delta_{2}}{\Gamma}=5$. (b) Attractive photons: bound state energies (solid) become resonant with energies of incoming photons (dashed) for specific values of $\kappa$. The error bars represent the decay rate of each bound state.

$\left(\alpha_{0} d\right)^{2} \ll 1$, the noise effect due to absorption can be ignored [27]. On the other hand, the two-photon state transmission is further suppressed due to an extra nonlinear phase shift that brings this state out of transmission resonance (for a similar effect in a cavity, see ref. [21]), which results in antibunching of the transmitted light $\left(g_{2}(0)<1\right)$. The antibunching implies that the transmitted field acquires nonclassical character [40], and the effect becomes more pronounced as OD is increased, which increases the effective system finesse (fig. 3(a)). Physically, this behavior occurs because the transmission of the single-photon component of the field remains intact while two-photon and higher components become strongly reflected, and thus it can be said that this system operates as a single-photon switch. Moreover, we observe a significant spatial deformation of the two-photon wave function inside the waveguide as $\kappa$ is increased, as seen in fig. 1. In particular, a cusp develops along the diagonal $z_{1}=z_{2}$ and the majority of the photon density lies in the off-diagonal regions, indicating that the two photons repel each other. In the limit of $\kappa \rightarrow \infty$, the diagonal elements must be completely suppressed which leads to perfect antibunching. Similar behavior involving the "self-organization" of photons in an NLSE system has been discussed in ref. [7].

In the case of attractive interaction $\left(\kappa<0\right.$, i.e., $\left.\Delta_{2}<0\right)$, the system exhibits a very different behavior from the repulsive case. In fig. 1, we plot $g_{2}(0)$ for the transmitted field $v s$. $\kappa d$. For small $|\kappa| d$, the nonlinear phase acquired by multi-photon components enhances (suppresses) their reflection (transmission), much like in the repulsive case. This is responsible for $g_{2}(0)$ dropping slightly below unity, despite the attractive interaction, and yields a weak singlephoton switching effect. At larger values of $|\kappa| d$, oscillations develop in the correlation function, yielding strong bunching behavior at particular values of $\kappa d$. A closer analysis reveals that the locations of these resonances correspond to resonant excitation of specific two-photon bound states, $2 \delta_{r e s}=E_{n}^{b}$. In particular, while the detuning 


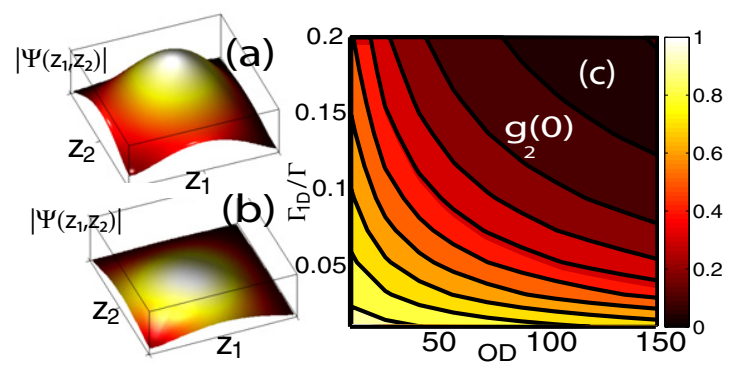

Fig. 4: (Colour on-line) Nonlinear absorption: two-photon wave function amplitude in the absence $\left(\Delta_{2} \gg \Gamma\right)$ (a) and presence $\left(\frac{\Delta_{2}}{\Gamma}=0\right)(\mathrm{b})$ of nonlinear absorption. The physical parameters are chosen such that $\mathrm{OD}=70, T \simeq 20 \%$ and $\frac{\Gamma_{1 D}}{\Gamma}=10 \%$. (c) Correlation function $g_{2}(\tau=0)$ of the transmitted light as a function of optical depth (OD) and single-atom cooperativity when the frequency is set to the single-photon transmission resonance with $T \simeq 20 \%$ and $\frac{\Delta_{2}}{\Gamma}=0$.

$\delta_{\text {res }}$ of the input field is fixed, the bound state energies $E_{n}^{b}$ vary quadratically with changing $\kappa$, causing the two to come into resonance at particular values of $\kappa$. This is shown in fig. 3(b), where the bound-state energies are evaluated via eq. (9). This effect is further confirmed by examining the two-photon wave function at each of these oscillation peaks (fig. 1), where the photon density becomes localized along the diagonal and indicates a bound state in the relative coordinates. Simultaneously, an increasing number of nodes and antinodes develop along the diagonal for increasing $|\kappa| d$, which are associated with the higher momenta of the center-of-mass motion. These resonances deviate significantly from the predictions of the semiclassical picture and also have no counterpart in cavity QED, due to the unique spatial degrees of freedom available in our system. Importantly for experiments, these bound states can be probed with classical light, simply by examining higher-order correlation functions in the output field. For example, the bunching peak associated with the second bound state at $|\kappa| d \simeq 5$, can be observed with parameter choices $T \simeq 1 \%$ and $\frac{\Delta_{2}}{\Gamma}=-5$, using an optical depth of $\mathrm{OD} \simeq 3500$ and coupling efficiency $\frac{\Gamma_{1 D}}{\Gamma}=0.2$.

Now we consider a system with purely nonlinear absorption ( $\kappa$ being imaginary). This situation corresponds to the case where the incoming photons are resonant with the atomic transition $|c\rangle \rightarrow|d\rangle$ that provides the nonlinearity, i.e., $\Delta_{2}=0$. Such a system can behave as an absorptive single-photon switch, which tends to destroy multiphoton components propagating through the fiber while the single-photon component is left intact. Figure 4(a),(b) shows the suppression of the two-photon states inside the medium, in the presence of the nonlinear absorption. Consequently, the transmitted field will be antibunched. Similar to the case of repulsive interaction in fig. 3(a), the antibunching behavior is more pronounced for larger coupling efficiency and optical depth. However, the nonlinear absorption effect on the correlation function is stronger compared to the repulsive interaction case for the same parameters, as shown in fig. 4(c). This is readily related to the fact that the effective nonlinearity $(|\kappa|)$ is stronger when photons are resonant with the nonlinear transition. As an example, for $\mathrm{OD}=200$, coupling efficiency $\frac{\Gamma_{1 D}}{\Gamma}=0.1, T \simeq 20 \%$, we obtain $g_{2}(0)=0.1$.

In conclusion, we have presented a theoretical approach to investigate transport properties of a few field quanta inside a one-dimensional, finite-size nonlinear medium obeying the NLSE. When the system is driven out of equilibrium with a coherent field at the boundary, the strong interaction inside the medium is manifested in the correlation functions of the transmitted field. In particular, for a repulsive interaction, the transmitted field is antibunched, while for an effective attractive interaction, the transmitted field can be either bunched or antibunched. Moreover, in the attractive case, we show that the bunching is due to the resonant excitation of photonic bound states. Recent progress in loading a large number of cold atoms into hollow-core optical fibers [4] should allow one to probe these effects in ongoing experiments.

We thank V. Gurarie, A. Sørensen and S. Fan for useful discussions. This work was partially supported by NSF, NSF DMR-0705472, Swiss NSF, CUA, DARPA, Packard Foundation and AFOSR-MURI. DEC acknowledges support from the Gordon and Betty Moore Foundation through Caltech's CPI, and NSF PHY-0803371.

Note added in proofs: Shortly after the initial submission of this paper, ref. [13] appeared on the arXiv which studies the dissipation-induced Tonks-Girardeau gas of polaritons.

\section{REFERENCES}

[1] Widera A., Trotzky S., Cheinet P., Fölling S., Gerbier F. and Bloch I., Phys. Rev. Lett., 100 (2008) 140401.

[2] Cronenwett S., Oosterkamp T. and Kouwenhoven L., Science, 281 (1998) 540.

[3] Zumbühl D., Marcus C., Hanson M. and Gossard A., Phys. Rev. Lett., 93 (2004) 256801.

[4] Bajcsy M., Hofferberth S., Balic V., Peyronel T., Hafezi M., Zibrov A. S., Vuletic V. and Lukin M. D., Phys. Rev. Lett., 102 (2009) 203902.

[5] Schnorrberger U., Thompson J. D., Trotzky S., Pugatch R., Davidson N., Kuhr S. and Bloch I., Phys. Rev. Lett., 103 (2009) 033003.

[6] Vetsch E., Reitz D., Sagué G., Schmidt R., Dawkins S. T. and Rauschenbeutel A., Phys. Rev. Lett., 104 (2010) 203603.

[7] Chang D. E., Gritsev V., Morigi G., Vuletic V., Lukin M. D. and Demler E. A., Nat. Phys., 4 (2008) 884.

[8] Angelakis D., Santos M. and Bose S., Phys. Rev. A, 76 (2007) 31805.

[9] Hartmann M. J., Brandao F. G. S. L. and Plenio M. B., Nat. Phys., 2 (2006) 849. 
[10] Greentree A. D., Tahan C., Cole J. H. and Hollenberg L. C. L., Nat. Phys., 2 (2006) 856.

[11] Cho J., Angelakis D. and Bose S., Phys. Rev. Lett., $101(2008) 246809$.

[12] Carusotto I., Gerace D., Tureci H., Liberato S. D., Ciuti C. and ImamoĞlu A., Phys. Rev. Lett., 103 (2009) 033601.

[13] Kiffner M. and Hartmann M., Phys. Rev. A, 81 (2010) 021806(R).

[14] Ghosh S., Sharping J. E., Ouzounov D. G. and Gaeta A. L., Phys. Rev. Lett., 94 (2005) 093902.

[15] Kien F. L. and Hakuta K., Phys. Rev. A, 77 (2008) 033826.

[16] Birnbaum K. M., Boca A., Miller R., Boozer A. D., Northup T. E. and Kimble H. J., Nature, 436 (2005) 87.

[17] Shen J.-T. and FAn S., Phys. Rev. Lett., 98 (2007) 153003.

[18] Chang D. E., Sørensen A. S., Demler E. A. and Lukin M. D., Nat. Phys., 3 (2007) 807.

[19] Agrawal G. P., Nonlinear Fiber Optics (Academic Press, New York) 2007.

[20] Drummond P. D., Shelby R. M., Friberg S. R. and Yамамото Y., Nature, 365 (1993) 307.

[21] Imamoglu A., Schmidt H., Woods G. and Deutsch M., Phys. Rev. Lett., 79 (1997) 1467.

[22] Lieb E. H. and Liniger W., Phys. Rev., 130 (1963) 1605.

[23] Korepin V. E., Bogoliubov N. M. and Izergin A. G., Quantum Inverse Scattering Method and Correlation Functions (Cambridge University Press) 1993.
[24] Kinoshita T., Wenger T. and Weiss D., Science, 305 (2004) 1125.

[25] Parades B. et al., Nature (London), 429 (2004) 277.

[26] Calabrese P. and Caux J.-S., Phys. Rev. Lett., 98 (2007) 150403.

[27] Hafezi M., Chang D., Gritsev V., Demler E. and Lukin M., arXiv:0911.4766 (2009) http://lanl.arxiv. org/abs/0911.4766.

[28] Girardeau M., J. Math. Phys., 1 (1960) 516.

[29] André A. and Lukin M. D., Phys. Rev. Lett., 89 (2002) 143602.

[30] BajCSy M., Zibrov A. S. and Lukin M. D., Nature, 426 (2003) 638.

[31] André A., Bajcsy M., Zibrov A. S. and Lukin M. D., Phys. Rev. Lett., 94 (2005) 063902.

[32] Schmidt H. and Imamoglu A., Opt. Lett., 21 (1996) 1936.

[33] Jona-Lasinio M., Morsch O. and Cristiani M., Phys. Rev. Lett., 91 (2003) 230406.

[34] Wimberger S., Mannella R. and Morsch O., Phys. Rev. Lett., 94 (2005) 130404.

[35] Rapedius K. and Korsch H. J., Phys. Rev. A, 77 (2008) 063610.

[36] Lai Y. and Haus H., Phys. Rev. A, 40 (1989) 854.

[37] Bloch I., Dalibard J. and Zwerger W., Rev. Mod. Phys., 80 (2008) 885.

[38] Astrakharchik G., Boronat J., Casulleras J. and Giorgini S., Phys. Rev. Lett., 95 (2005) 190407.

[39] Batchelor M., Bortz M., Guan X. W. and Oelkers N., J. Stat. Mech.: Theory Exp. (2005) L10001.

[40] Mandel L., Phys. Scr., T12 (1986) 34. 